Trauma Berufskrankh $2012 \cdot$ 14[Suppl 3]:271-275 DOI 10.1007/s10039-012-1853-1

Online publiziert: 25. Mai 2012

(c) Springer-Verlag 2012

\section{R. Nieper}

Klinikverbund der gesetzlichen Unfallversicherung e. V., Berlin

\section{Im Verbund noch stärker}

\section{Klinikverbund der gesetzlichen Unfallversicherung e. V. (KUV)}

KUV

Klinikverbund der gesetzlichen Unfallversicherung

Abb. $1 \Delta$ LOGO des KUV

Der Klinikverbund der gesetzlichen Unfallversicherung e. V. (KUV, - Infobox 1, - Abb. 1) wurde am 16.09.2010 gegründet. Die Grundlagen für den organisierten kollegialen Austausch sowie die $\mathrm{Zu}$ sammenarbeit der BG-Kliniken (BG: Berufsgenossenschaft) untereinander wurden jedoch bereits durch die Vereinigung der Berufsgenossenschaftlichen Kliniken (VBGK) gelegt. Ein wesentlicher Anstoß für den Schritt zum Verbund lag daher nicht zuletzt im Konsens über den qualitativen Nutzen, den die Kliniken aus der Integration in eine engere Verbundstruktur ziehen können. Gleichzeitig reagierte man mit diesem Zusammenschluss auf die sich abzeichnende Entwicklung eines neuen berufsgenossenschaftlichen Heilverfahrens. Die Gründung des Verbundes hat damit auch eine klare politische Dimension.

Der Autor des vorliegenden Beitrags, Reinhard Nieper, ist seit 01.07.2011 Geschäftsführer des KUV und führte in den ersten Wochen seiner Tätigkeit eine umfassende Bestandsaufnahme durch. Auf dieser Grundlage wurden Handlungsfelder und Ziele für den Klinikverbund definiert und auf der 34. Unfallmedizinischen Tagung in Baden-Baden am 22.09.2011 vorgestellt. Der vorliegende Beitrag stellt eine stellenweise aktualisierte Fassung dieses Vortrags dar.

\section{Vier Erfolgsgaranten}

Grundsätzlich ist festzustellen, dass sich der Klinikverbund in einer ausgesprochen positiv zu bewertenden Ausgangslage befindet. Er kann sich dabei m. E. auf 4 solide, den Erfolg garantierende Pfeiler stützen:

Integrativer Ansatz. Dieser spiegelt sich in der Sektoren übergreifenden Versorgung (Akut- und Rehabilitationsmedizin) wider.

SGB-VII-Grundsatz (SGB: Sozialgesetzbuch). Nach diesem Grundsatz sind, im Unterschied zum SGB V, die Gesundheit und Leistungsfähigkeit nach dem Eintritt eines Arbeitsunfalls oder einer Berufskrankheit mit allen geeigneten Mitteln wiederherzustellen ( $\$ 1$ Ziffer 2, SGB VII). Daraus folgt, dass der Ebene der qualitativen Leistungserbringung, im Vergleich zur Ökonomieebene, eine übergeordnete Bedeutung zukommt.

Verpflichtung zur Gemeinnützigkeit. Sie ergänzt vorgenannten Grundsatz und unterstreicht eine Unternehmenspolitik die nicht auf Profitmaximierungen und Share-Holder-Value fokussiert, sondern bei der jeder erwirtschaftete Euro in den Kliniken verbleibt. Die Einrichtungen des Klinikverbunds arbeiten wirtschaftlich, um Menschen in Not zu behandeln, und behandeln nicht Menschen in Not, um Gewinne zu generieren.

\begin{tabular}{|l|}
\hline Infobox 1 Kontakt \\
\hline Klinikverbund der Gesetzlichen \\
Unfallversicherung e.V. (KUV) \\
http://www.k-uv.de \\
info@klinikverbund-uv.de \\
\hline
\end{tabular}

Selbstverwaltung. Schließlich arbeitet der KUV nach den Prinzipien der Selbstverwaltung und grenzt sich damit, insbesondere durch das Element der demokratisch-partizipativen Führung, von Organisationsformen mit klassisch hierarchischen Strukturen ab.

\section{Spitzenleistungen}

Die genannten Erfolgsgaranten bilden weitestgehend den Hintergrund, vor welchem sich die herausragende Rolle abzeichnet, die die BG-Kliniken bei der Akutversorgung von Schwerstverletzten spielen. Die Kliniken können dabei auf exzellente Reputationen insbesondere bei der Behandlung von Polytraumen, Schädel-Hirn-Verletzungen, Rücken-, Handund Schwerbrandverletzten sowie in der septischen Chirurgie verweisen, die über die nationale Fachwelt hinausgehen. Das korrespondiert mit einer entsprechend ausgeprägten Behandlungskomplexität in den BG-Kliniken. Der Case-Mix-Index (CMI), der hierfür als Indikator herangezogen werden kann, weist für die BG-Kliniken in 2010 einen Mittelwert von 1,61 aus und liegt damit deutlich über dem bundesdeutschen Durchschnitt für 2010 $(1,08,[1])$. 
Der überdurchschnittliche Schweregrad der zu behandelnden Patienten weist zugleich auf entsprechend hohe Anforderungen hin, die an jede einzelne Mitarbeiterin und jeden einzelnen Mitarbeiter in den Häusern gestellt werden. Wenn man sich einer Aufgabe wie der Versorgung von Schwerstverletzten verpflichtet fühlt, bedarf es außerordentlicher Leistungsbereitschaft, um nicht zu sagen Spitzenleistung, wie sie für alle BG-Kliniken kennzeichnend ist. Nationales wie internationales Ansehen in Fachkreisen, Spitzenleistungen in den Kliniken sowie ein ausgeprägter gemeinnütziger Charakter verdichten sich in einer entsprechend positiven kulturellen Identität der BG-Kliniken. Der Wert dieses Identifizierungspotenzials, das die BGWelt - trotz aller Meinungsvielfalt - ihren Mitarbeiterinnen und Mitarbeitern bietet, zeigt sich immer dort, wo es zu einer Positionierung gegenüber Dritten oder Fremdorganisationen kommt.

Diese „soft facts“ sind zwar nicht direkt formalisierbar, aber für den Erfolg des Verbunds gleichwohl von hoher Relevanz. Es ist daher unerlässlich, sie auch in Zukunft zu stärken und gegenüber der Fachwelt und Öffentlichkeit kommunikativ noch deutlicher in den Vordergrund zu rücken.

\section{Spannungsfelder}

\section{Standortentwicklung der Kliniken vs. Verbundorientierung}

Dem KUV bieten sich, wie oben ausgeführt, sehr günstige Startbedingungen. Die Arbeit an gewachsenen Strukturen erzeugt jedoch zwangsläufig auch Spannungsfelder. Im konkreten Fall resultieren diese aus der Kollision zwischen einem etablierten autonomen Handlungsverständnis einzelner Trägervereine und den mit der Satzung verbindlich definierten Aufgaben und Kompetenzen des Verbunds. Die Verantwortungsträger in den Kliniken verweisen an dieser Stelle auf die inhärente Gefahr einer möglichen Einengung des eigenen Handlungs- und Entscheidungsspielraums, ausufernde administrative Prozeduren sowie die Ausweitung zentraler Einflussnahmen. Gleichwohl ist unbestritten, dass die überragen- de Stellung der Kliniken in einem sich weiter verdichtenden Krankenhausmarkt nur durch eine abgestimmte, einheitliche Verbundstruktur gewährleistet werden kann.

\section{Zwischen Unternehmen und Institution}

Aus Obigem ergibt sich indirekt eine weitere Frage und damit ein weiteres Spannungsfeld, welches sich im Übrigen auch KUV-intern widerspiegelt: Die Frage, in welcher Form sich der Klinikverbund am Markt zu platzieren hat, ruft bei den beteiligten Akteuren z. T. stark voneinander abweichende Antworten hervor. Diese Divergenzen können darauf zurückgeführt werden, dass die BG-Kliniken einerseits strukturell in behördenähnliche Abläufe eingebunden sind, gleichzeitig aber eine aktive Rolle auf dem Krankenhausmarkt spielen sollen. Hier zeichnet sich sozusagen ein Hin und Her zwischen institutionellem vs. unternehmerischem Selbstbild ab. Auf der einen Seite steht dabei mit der Gründung des KUV der Wunsch nach Etablierung von Managementstrukturen im Raum. Auf der anderen Seite existiert das Selbstbild der BG-Klinik als Institution, für die die Mechanismen des Marktes keine Geltung haben, da sie einzig dem Primat der Politik untergeordnet ist.

\section{Differenzierung in der medizinischen Leistungserbringung}

Ein weiteres Spannungsfeld ergibt sich ganz konkret aus dem Klinikalltag. Behandelnde Mediziner, Pflegekräfte und Therapeuten stehen hier vor einem Konflikt, der sich aus den unterschiedlichen Leistungen, die den Patienten je nach Versicherungsfall zukommen, ergibt. Unterschiedliche Finanzierungsformen der Behandlungen von GKV- (gesetzliche Krankenversicherung) und PKV-Patienten (PKV: private Krankenversicherung) gegenüber BG-Patienten korrespondieren zwangsläufig mit unterschiedlichen Behandlungsformen und -intensitäten und stellen die am Heilungsprozess beteiligten Akteure vor nicht zu unterschätzende moralisch-ethische Probleme.
Dieses Dilemma hat seinen Ursprung in den der Öffentlichkeit wenig bekannten, unterschiedlichen gesetzlichen Grundlagen zwischen der KV und der Unfallversicherung. Während Erstere ein Produkt des gesellschaftlichen Solidaritätsprinzips ist, welches wirtschaftlichen Limitierungen unterliegt, kommt bei Letzterer ein Schadensersatzanspruch zur Geltung (Haftungsablösung), den der Versicherte gegenüber den Unfallversicherungsträgern hat. Im Kern stehen sich hier Solidaritätsprinzip und Schadensersatzforderung gegenüber.

\section{Mehrere Rollen der Unfallversicherungsträger}

Mit der Frage der Vergütung steht gleichzeitig die nach der Rolle, die die Unfallversicherungsträger für die Kliniken innehaben, im Raum. Bedingt durch ihre strukturelle Vielfalt treten die Berufsgenossenschaften im gegenwärtigen Szenario in unterschiedlichsten Funktionen und Rollen in Erscheinung. Sie haben bezüglich der Kliniken sowohl eine eigentümerähnliche Rolle inne, sind gleichzeitig aber auch Kostenträger. Sie sind somit sowohl Kunde als auch Partner.

So unterschiedlich wie die Kliniken die Rolle der Berufsgenossenschaften bewerten, so unterschiedlich sind auch die Verhaltensweisen im Umgang mit diesen. Wenngleich die Besonderheit des KUV darin besteht, dass über die gleichmäßige Verteilung der Stimmrechte zwischen Unfallversicherungsträgern und Trägervereinen eine gleichberechtigte Teilhabe beider Seiten an den Entscheidungsprozessen sichergestellt ist, hat der Verbund seine eigene Position in dieser Frage noch zu bestimmen. Diese Unbestimmtheit in Bezug auf die jeweiligen Rollen spiegelt sich auch in dem Umstand wider, dass man sich bisher weder in dokumentierter noch in inhaltlicher Form auf ein konsentiertes Unternehmensziel festlegte. Es wird also eine zeitnah zu klärende Frage sein, wie eine BG-Klinik strukturiert sein soll. Bis zur genaueren Festlegung sollten sich jedoch die Handlungsprämissen auf 2 Punkte konsentieren lassen: Einerseits muss berücksichtigt werden, dass den Anforderungen, die die Unfallversicherungsträger an die Kliniken stellen, vorrangig 
nachgekommen wird. Seitens der BGKliniken gilt es hier zu erkennen, dass sie ein Akteur in einem Netzwerk sind, das in seiner Gesamtheit das umfassende berufsgenossenschaftliche Heilverfahren widerspiegelt und dessen Ziel es ist, eine zügige und nahtlose Rehabilitation der Verletzten sicherzustellen. Andererseits sollen und dürfen die Einrichtungen am Gesundheitsmarkt teilnehmen. Denn nur so kann die wirtschaftliche und qualitative Spitzenstellung, die letztlich wieder den Anforderungen der Unfallversicherungsträger dient, sichergestellt werden.

\section{Handlungsfelder}

Solche Spannungsfelder wie oben beschrieben dürfen aber keinesfalls als nicht zu überwindende Hindernisse oder Gräben missinterpretiert werden. Sie zeigen vielmehr Potenziale auf, aus denen sich entsprechende Handlungen ableiten müssen. Der Klinikverbund wurde daher in den vergangenen Wochen hinsichtlich der in seiner Satzung formulierten Zielvorstellungen analysiert. Die Resultate wurden anschließend mit den Zielvorgaben der vorab definierten Geschäftsbereiche Kunden/Markt, Personal/Kommunikation, kaufmännische/technische Infrastruktur, Finanzierung/Controlling und Qualität/Prozesse verglichen. Die im Folgenden aufgeführten Handlungsfelder sind das vorläufige Ergebnis dieser Anstrengungen.

\section{Kunden/Markt}

Es muss deutlicher herausgehoben werden, dass mit dem Verbund eine führende Stellung am Markt angestrebt wird. Eine Voraussetzung dafür ist, dass man sich dezidiert mit den Produktlinien bzw. einer Definition derselben auseinandersetzt. Das bedeutet aber nicht, dass unterschiedliche Häuser nicht auch unterschiedliche Produkte darstellen können. Eine Abstimmung hinsichtlich der Produktlinien ist aus BG-Perspektive jedoch unerlässlich. So sollten beispielsweise Kliniken und Unfallversicherungsträger gemeinsam einen verbindlichen Rahmen für eine effiziente und bedarfsgerechte Heilbehandlung und Rehabilitation schwerer Unfallverletzungen definieren,

Trauma Berufskrankh 2012 · 14[Suppl 3]:271-275 DOI 10.1007/s10039-012-1853-1

(c) Springer-Verlag 2012

\section{R. Nieper \\ Im Verbund noch stärker. Klinikverbund der gesetzlichen Unfallversicherung e. V. (KUV)}

\section{Zusammenfassung}

Der Klinikverbund der gesetzlichen Unfallversicherung e. V. (KUV) wurde am 16.09.2010 gegründet. Im Sommer 2011 wurde eine umfassende Bestandsaufnahme durchgeführt, auf deren Grundlage Handlungsfelder und Ziele für den KUV definiert wurden. Dessen Ausgangslage ist dabei ausgesprochen positiv zu bewerten, wobei 4 Faktoren, der integrative Ansatz, der SGB-VII-Grundsatz (SGB: Sozialgesetzbuch), die Verpflichtung zur Gemeinnützigkeit und die Prinzipien der Selbstverwaltung, eine wichtige Rolle spielen. Doch es gibt auch Spannungsfelder, die keinesfalls als nicht zu überwindende Hindernisse missinterpretiert werden dürfen, sondern vielmehr Potenziale aufzeigen, aus denen sich entsprechende Handlungen ableiten müssen.
So muss z. B. im Bereich Kunden/Markt deutlicher herausgehoben werden, dass mit dem Verbund eine führende Stellung am Markt angestrebt wird. Auf kaufmännisch/technischem Bereich wäre es sinnvoll, durch Prozessoptimierungen die Investitionsfähigkeit der Häuser zu sichern. Auch die Bereiche Personal/Kommunikation, Finanzierung/Controlling und v. a. Qualität/Prozesse können noch optimiert werden. Diese ersten Handlungsfelder müssen nun in konkrete Konzepte überführt und umgesetzt werden.

\section{Schlüsselwörter}

Kunden/Markt · Personal/Kommunikation . Kaufmännische/Technische Infrastruktur . Finanzierung/Controlling · Qualität/Prozesse

\section{Stronger in an alliance. Klinikverbund der gesetzlichen Unfallversicherung e. $V$. (Federation of Statutory Accident Insurance Institutions)}

\section{Abstract}

The Klinikverbund der gesetzlichen Unfallversicherung e. V. (KUV, Federation of Statutory Accident Insurance Institutions) was established on 16 September 2010. Based on comprehensive stocktaking carried out in the summer of 2011, the fields of activity and goals of the KUV were defined. Its starting position can be assessed as extremely positive and four factors play an important role: the integrative approach, the Code of Social Law VII policy, the commitment to public benefit, and the principles of self-administration. However, there are also areas of conflict that on no account should be misinterpreted as insurmountable obstacles, but rather serve to identify potential options to devise appropriate courses of action. For example, in the realm of consumer/market it must be clearly emphasized that the aim of the alliance is to obtain a leading position in the marketplace. In the commercial/technical domain it would be expedient to solidify the investment capabilities of the institutions by process optimization. The areas of personnel/ communication, financing/controlling, and especially quality/processes can also be further optimized. These first fields of activity must now be converted to tangible concepts and implemented.

\section{Keywords}

Consumer/market · Personnel/ communication · Technical/commercial infrastructure · Financing/controlling · Quality/ processes an dem sich andere Einrichtungen orientieren können. Gleiches gilt für die Entwicklung und den Aufbau von Qualitätssicherungsstrukturen, in die auch andere, an der Schwerstverletztenversorgung beteiligte Einrichtungen eingebunden werden könnten. Über diesen Weg könnte das oben erwähnte Spannungsfeld der differenzierten medizinischen Leistungserbringung bei der Behandlung von GKV/
PKV- bzw. BG-Patienten einer sachorientierten Lösung zugeführt werden.

\section{Personal/Kommunikation}

In diesem Bereich kann an die erfolgreiche Arbeit des VBGK angeknüpft und darüber hinaus auf das erwähnte hohe Ansehen der berufsgenossenschaftlichen Kliniken in der Fachwelt zurückgegrif- 
fen werden. Dank dieser guten Reputation stellt die zunehmend schwerer werdende Rekrutierung von ärztlichem und pflegerischem Fachpersonal für die BGKliniken, im Vergleich zu anderen Häusern, ein bislang geringeres Problem dar. In Hinblick auf die Erhaltung der Zukunftsfähigkeit ist es dennoch ratsam, bereits heute über entsprechende Personalentwicklungsprogramme nachzudenken.

Die Optimierung der internen und externen Kommunikation stellt ein weiteres Handlungsfeld in diesem Bereich dar. Auch hier kann das Image der regional hoch angesehenen Kliniken ein Ausgangspunkt für eine gemeinsame Markenbildung sein. Auch allein schon hinsichtlich der vorgenannten Aspekts der Personalakquise ist ein gemeinsames Marketing sinnfällig und unerlässlich.

\section{Kaufmännische/technische Infrastruktur}

Der Erfolg der jeweiligen Kliniken liegt ganz erheblich auch darin begründet, dass sie auf regionaler Ebene in verschiedene Netzwerke und Kooperationen eingebunden sind. Zwischen einzelnen Einrichtungen der BG-Kliniken ist dieses Kooperationsverhältnis jedoch ausbaufähig. Es gibt eine Reihe von Bereichen, in denen es sinnvoll sein kann, Synergien zu schaffen. Ziel dieser Maßnahmen muss es sein, durch Prozessoptimierungen die Investitionsfähigkeit der Häuser zu sichern. Ich könnte mir vorstellen, dass die Häuser hier ihr Potenzial teilweise noch nicht vollständig ausgeschöpft haben und es hinsichtlich der Wirtschaftlichkeit einzelner Häuser noch Spielräume gibt. Synergien lassen sich vor allen Dingen auch unter dem Aspekt des Wissenstransfers schaffen. Der Klinikverbund wird es sich auf Dauer nicht leisten können, mit erheblichem Aufwand und unter hohem Ressourceneinsatz entwickeltes Knowhow nicht allen Teilnehmern gleichermaßen zur Verfügung zu stellen. Dieser Schatz muss entdeckt und gehoben werden.

\section{Finanzierung/Controlling}

Die finanzielle Handlungsfähigkeit ist im Kern gegeben, kann aber noch optimiert werden. Ein wesentlicher, unter dem Stichwort Kostentransparenz subsumierbarer Bestandteil dieses Optimierungsprozesses wird ein leistungsabbildendes Finanzierungssystem sein.

Gegenüber den Unfallversicherungsträgern wird es mittel- und langfristig nicht mehr vertretbar sein, dass über tagesgleiche Pflegesätze Kosten bei den Unfallversicherungsträgern verursacht werden, die für diese nicht transparent nachvollziehbar sind. Konsens besteht außerdem darin, dass neben diesen vergleichbaren Abrechnungsgrößen zu anderen Krankenhäusern v. a. fünf weitere Faktoren in einem neuen Vergütungssystem als Kosten erhöhend zu berücksichtigen sind:

- der Anspruch auf höhere Qualität (z. B. durch Weiterbildung und Forschung),

- die z. T. nicht exakt abgrenzbare integrierte Rehabilitation,

- die durch die notwendigen technischen Besonderheiten entstehenden höheren Instandhaltungskosten,

- die im Vergleich zu Krankenhäusern mit der Möglichkeit zur Mischfinanzierung höheren Vorhaltekosten, die sich aus der spezifischen Leistungsstruktur der BG-Kliniken notwendigerweise ergeben, sowie

- die besonderen Dienstleistungen, die die Kliniken für die Unfallversicherungsträger erbringen und die finanziell abgebildet werden müssen.

\section{Qualität/Prozesse}

Dieser Bereich stellt das für die Daseinsberechtigung und Zukunftsfähigkeit der BG-Kliniken zentrale Arbeitsfeld dar. Dies leitet sich daraus ab, dass sich die Qualität der medizinischen und rehabilitativen Maßnahmen in den Häusern, die den Grundpfeiler des berufsgenossenschaftlichen Heilverfahrens bilden, gegenwärtig nicht valide und in der notwendigen Detailtiefe belegen lässt. Dies ist aus zweierlei Gründen jedoch zwingend erforderlich: Einerseits kann damit in internen Diskussionen kritischen Nachfragen standgehalten werden. Andererseits, und das ist von besonderer Wichtigkeit, sichert nur eine Evaluation von Qualitätsstandards und Prozessabläufen die Möglichkeit, das gesamte System der BG-Kli- niken und damit das der Unfallversicherungsträger sowohl gegenüber Dritten als auch gegenüber der kritischen Öffentlichkeit, rechtfertigen zu können.

\section{Beteiligungsstruktur des KUV}

Diese ersten Handlungsfelder müssen nun in konkrete Konzepte überführt werden. Deren Erarbeitung folgt einem Regelkreis, der sich wiederum in eine Arbeits-, eine Strategie- und eine politische Ebene unterteilen lässt.

Auf der Arbeitsebene bleibt die bisherige VBGK-Struktur der Arbeitsgruppen im Wesentlichen unverändert. Sie unterscheidet sich nur insofern, dass sie nicht wie bisher von den Geschäftsführern einzelner Einrichtungen, sondern von einem der Bereichsleiter des KUV geleitet wird. Die Strategieebene erhält mit den neu eingerichteten Strategiegruppen eine erweiterte Struktur. Diese Strategiegruppen orientieren sich schematisch an den 5 vorgenannten Bereichen und werden jeweils durch den Geschäftsführer des KUV sowie einen der jeweiligen Bereichsleiter des KUV und 2 bis 3 Geschäftsführern der Klinikträgervereine besetzt. Ihre Aufgabe ist es, die Verbindung zwischen der Fachebene der Arbeitsgruppen und der politischen Ebene, in Form der Geschäftsführerkonferenz, zu gewährleisten. Die bisherige Arbeitsgruppe der Ärztlichen Direktoren wird zum Medizinischen Beirat und erfährt dadurch eine Aufwertung. Er wird zudem eng mit der Strategiegruppe Qualität/Prozesse zusammenarbeiten. Ein Vertreter der Ärztlichen Direktoren wird darüber hinaus Mitglied der Geschäftsführerkonferenz werden. Entscheidend ist, dass sämtliche Vorlagen, die im KUV-Vorstand vorgelegt werden, in der Geschäftsführerkonferenz besprochen werden. Diese erstellt jedoch lediglich ein Votum für den Geschäftsführer des KUV hinsichtlich der Kommunikation gegenüber den Unfallversicherungsträgern und hinsichtlich der Vorlage in den Vorstand des KUV. Die Entscheidungen des KUVVorstandes werden anschließend in die Trägervereine zurückgespielt.

Von zentraler Bedeutung für den Erfolg des KUV ist die verpflichtende Umsetzung dieser Entscheidungen. Den Arbeitsgruppen fällt dann wiederum die 
Dokumentation hinsichtlich der Umsetzung der Vorstandsentscheidungen in den einzelnen Einrichtungen zu. Damit ist ein geschlossener und zielführender Regelkreis geschaffen, dessen Errichtung, Umsetzung und Ausgestaltung Aufgabe des KUV in den kommenden Wochen und Monaten sein wird.

\section{Korrespondenzadresse}

R. Nieper
Klinikverbund der gesetzli-
chen Unfallversicherung e. V.,

Interessenkonflikt. Der korrespondierende Autor gibt an, dass kein Interessenkonflikt besteht.

The supplement containing this article is not sponsored by industry.

\section{Literatur}

1. Statistisches Bundesamt (2011) Gesundheit - Fallpauschalenbezogene Krankenhausstatistik (DRGStatistik) - Diagnosen, Prozeduren, Fallpauschalen und Case Mix der vollstationären Patientinnen und Patienten in Krankenhäusern. Fachserie 12, Reihe 6.4, 569 